\title{
New Trends in Immunology Membrane Biophysics
}

\author{
Santos Filho SD1,2,3* \\ ${ }^{1}$ Biosciences Department, Universidade Federal do Rio Grande do Norte, Brasil \\ ${ }^{2}$ Biophysical and Biometric Department, Universidade do Estado do Rio de Janeiro, \\ Brasil \\ ${ }^{3}$ Physiotherapy, FANEC/ UNIP, Brasil
}

\section{Review Article}

Volume 4 Issue 4

Received Date: June 21, 2019

Published Date: July 08, 2019

DOI: 10.23 880/ijbp-16000166

*Corresponding author: Sebastião David Santos-Filho, UERJ - Universidade do Estado do Rio de Janeiro, Instituto de Biologia Roberto Alcantara Gomes (IBRAG), Pavilhão Américo Piquet Carneiro (PAPC), Boulevard 28 de Setembro, 87, 20551-030, Rio de Janeiro - RJ, Brasil, Email: santos-filho@uerj.br

\section{Abstract}

Membranes are not limited to the cell surface; they are present throughout the cell interior, providing the basic structure of organelles. Membranes act as barriers to the free diffusion of various substances, including ions. Antibiotics transport through the membrane and the interactions between antigens and receptors of the immune system are enhanced by simultaneous interactions between lymphocyte-expressed co-receptors and molecules on antigen-presenting cells or on complex antigens. The type of antigens receptor present on $\mathrm{B}$ and $\mathrm{T}$ cells are a membrane-bound form of the immunoglobulin super-family molecules. I pretend in this work to present this membrane proteins and their function in the cellular body.

Keywords: Membrane; Antibiotics; Antigens; Receptors; Immunology; Biophysics

\section{Introduction}

Membranes are not limited to the cell surface; they are present throughout the cell interior, providing the basic structure of organelles. The first observation on the diffusion-limiting properties of the cell surface were made in the mid-nineteenth century by Nageli, who noted that cell surface acts as a barrier to free diffusion of dyes into the cell from the extracellular fluid. From this he deduced the presence of a plasma membrane. Later, Pfeffer drew a parallel between the osmotic properties of artificial semipermeable membranes and osmotic properties of living cells. This further proved that living tissues obey the laws of physics. Using red blood cells, Overton discovered that there is a close relationship between the lipid solubility of a substance and its ability to penetrate the cell membrane. He proved that high lipid solubility allows a substance to penetrate the cell membrane more rapidly. With the advent of electron microscopy, structural details of plasma membrane came to light. Robertson visualized the plasma membrane as dark-lightdark profile under electron microscope.

With modern separation techniques, it became possible to analyse the components of the membranes. This led to the discovery of artificial membranes. Later, many transport processes were investigated across these man-made membranes. Transport across the membranes is governed by physical and physics-chemical principles [1].

Membranes regulate the cytoplasmic concentration of dissolved ions and molecules rather precisely, establishing an intracellular milieu conducive to the finely 


\section{International Journal of Biochemistry \& Physiology}

balanced metabolic and synthetic activities of the cell. Membranes are specialized in different ways to perform special functions. For example, the membranes of nerve and muscle cells are specialized for the conduction of bioelectric impulse. Gross alterations of membrane structure can affect water balance within the cell. Specified deficiencies or alterations of certain membrane components lead to a variety of diseases [2].

\section{Membrane Structure}

All the membranes have a common molecular organization, i.e., under electron microscope they appear as three-layered structure. This three-layered structure consists of electron transparent region surrounded by electron dense lines on both the sides. The ubiquity of this trilaminar structure is termed as unit membrane. Membranes contain lipids, proteins and small amounts of carbohydrates. The unit membrane hypothesis according to which membrane proteins form continuous bilayer on lipid bilayer was modified by Singer, who suggested that proteins penetrate deeply into or through the lipid bilayer to form a 'mosaic'. The mosaic model was further modified to fluid mosaic model by Singer and Nicolson in 1972. As per this model, lipid phase of the membrane exists as a two-dimensional liquid, in which both protein and lipid molecules can diffuse. These form the basic structure of the membrane. The hydrophobic tails of the fatty acyls form the middle of the bilayer. Polar hydrophobic heads of the phospholipids line both surfaces. Integral proteins: These are embedded in the lipid bilayer where they interact with the fatty acyl chains. Peripheral proteins: These are associated with the membrane by specific protein-protein interactions. Oligosaccharides: They are bound to the membrane proteins, forming glycoproteins. Some of the oligosaccharides are bound to lipids [3,4] (Figure 1).

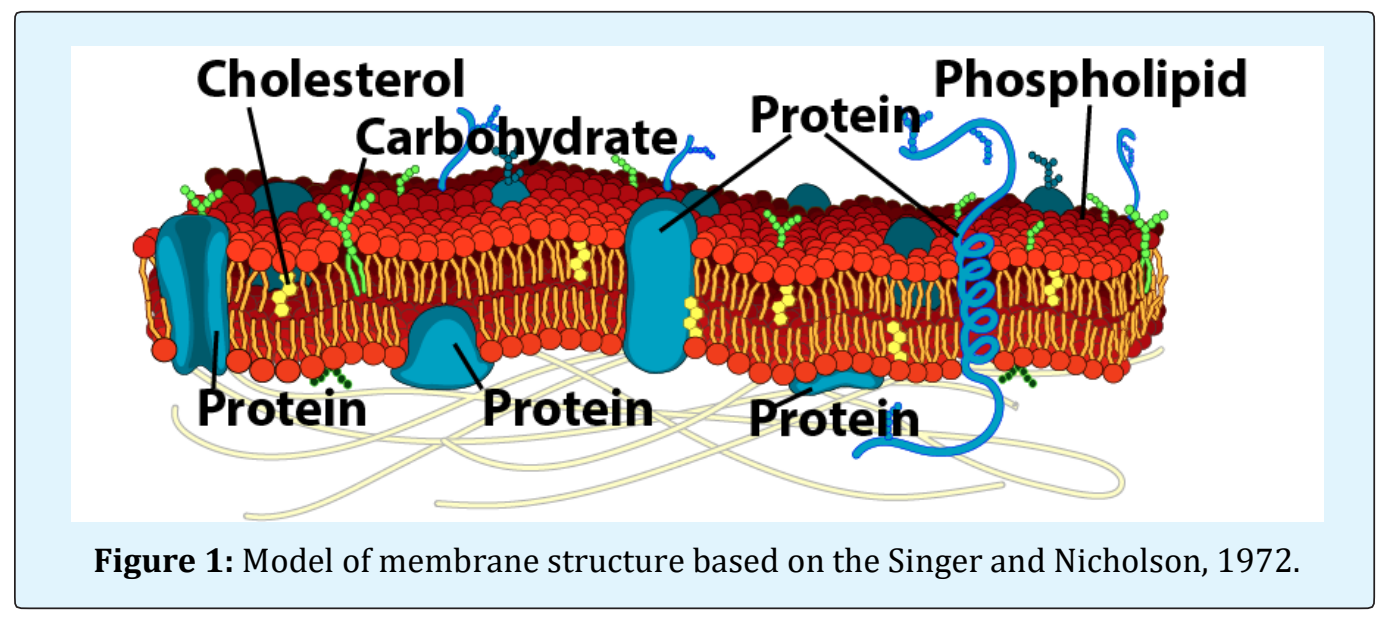

\section{Membrane Proteins}

Some of the proteins can be removed from membranes by agents that disrupt ionic and polar bonding, e.g., urea or high concentrations of salt solution. These are known as extrinsic or peripheral proteins. Other proteins, called intrinsic or integral proteins can be removed only by treating the membranes with detergents or with organic solvents. The peripheral proteins exist on the surfaces of the bilayer and are attached via ionic and polar bonds to the polar heads of the lipids or the integral proteins. The integral proteins are deeply imbedded in the bilayer and are anchored in the membrane by van der Waals bonds and hydrophobic interactions. Some of the integral proteins completely span the bilayer. If the integral proteins completely spanning bilayer exist in pair, a pore or protein channel is formed. Protein channels are watery pathways [4]. Each channel has definite diameter shape and electrical charge. Separate protein channels exist for sodium and potassium ions. These channels can be opened or closed by gates. Gates are gate-like extensions of channel proteins. Functionally, these channels can be divided into three types. One of this is an electoral or voltage stimulate channel, other is a chemical stimulated channel, and a physical way channel [5-8].

\section{Functions of Membrane Proteins}

Membrane proteins have functions according the structures they are present, some like this: 


\section{International Journal of Biochemistry \& Physiology}

I. They transport ions and molecules into and out of cells.

II. They receive signals from hormones and other chemicals in the surrounding fluid and transmit these signals in the cell interior.

III. Some of the peripheral proteins have contractile properties and their activation has been implicated in the rearrangement of membrane proteins which occur in response to various signals.

IV. Because some membrane-bound proteins are located on the outer faces of the cells. they are responsible for endowing cells with individuality that allow them to classify during differentiation and to form specific connections with other cells.

V. Certain membrane proteins act as enzymes which catalyse specific reactions of substrates in intracellular or extracellular fluid (like acetylcholine esterase).

VI. Some membrane proteins act as anchors for cytoskeletal components and for components of the extracellular matrix.

\section{Forces responsible for the Organization of Lipid Bilayer}

The primary physical forces responsible for organizing biological membrane are hydrophobic interactions between the fatty acyl chains of lipid molecules. These interactions result in the formation of a phospholipid bilayer. which is a sheet containing two layers of phospholipid molecules whose polar head groups face the surrounding water surface while the fatty acyl chains form a continuous hydrophobic interior.

\section{Glycocalyx}

The entire surface of the cell has a loose coat of carbohydrates. This is called glycocalyx. Carbohydrates are either bound to proteins, forming glycoproteins or to lipids, forming glycolipids. Carbohydrates help in attaching cell together. Since most of them are negatively charged. They impart overall negative surface charge, they also play important role in immune reactions [9] (Figure 2).

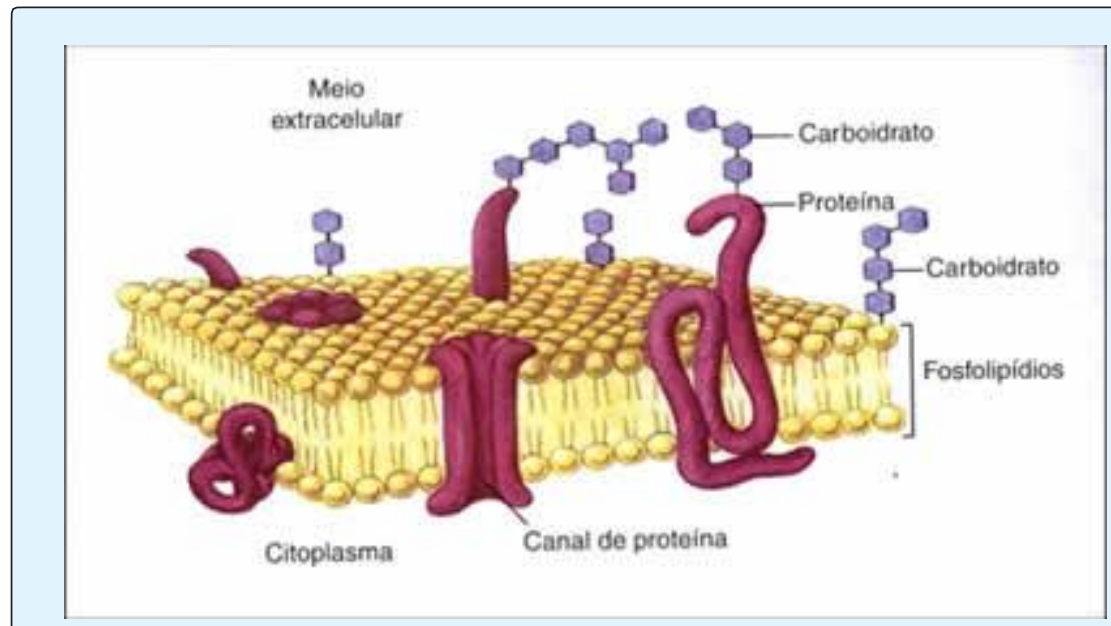

Figure 2: Membrane structure: the glicocalix.

\section{Asymmetry in Membranes}

Causes of asymmetry in the membranes are enumerated below:

I. Irregular distribution of proteins within the membrane.

II. Since different lipids have different charges and tend to contain different types of acyl-chain. The two halves of the bilayer could have different fluidities and surface charges.
III. There are regional asymmetries in membranes. These occur at villous border of mucosal cells, at gap junctions. light junctions and synapses.

IV. An inside-outside asymmetry of the membrane is due to external location of the carbohydrates attached to membrane proteins or due to presence of specific enzymes exclusively on outside or inside of membrane.

V. There is inside-outside (transverse) asymmetry of phospholipid molecules in the membrane. The 


\section{International Journal of Biochemistry \& Physiology}

choline-containing phospholipids are located mainly in the outer molecular layer; the amino phospholipids are preferentially located in the inner layer. Cholesterol is generally present in larger amounts outside than on the inside.

\section{Fluidity of the Membranes}

Cholesterol is the major determinant of membrane fluidity. Cholesterol increases the "micro-viscosity" in the interior of bilayer. In addition to this, cholesterol separates and disperses the tails of the fatty acyls. This causes the inner regions of the bilayer to become slightly more fluid. Presence of short fatty acyl chains, presence of double bonds in acyl chains, and temperature favour or fluid phase consistency. Lipids of the membrane show different types of movements within the membrane. It is very rapid and results in part from rapid thermal movements of fatty acyl chains, which promote intermolecular collisions [10]. Rotational motion it occurs about an axis perpendicular to the bilayer. Flip-flop motion Migration of lipid molecules from one monolayer: next results in flip-flop motion (Figure 3).

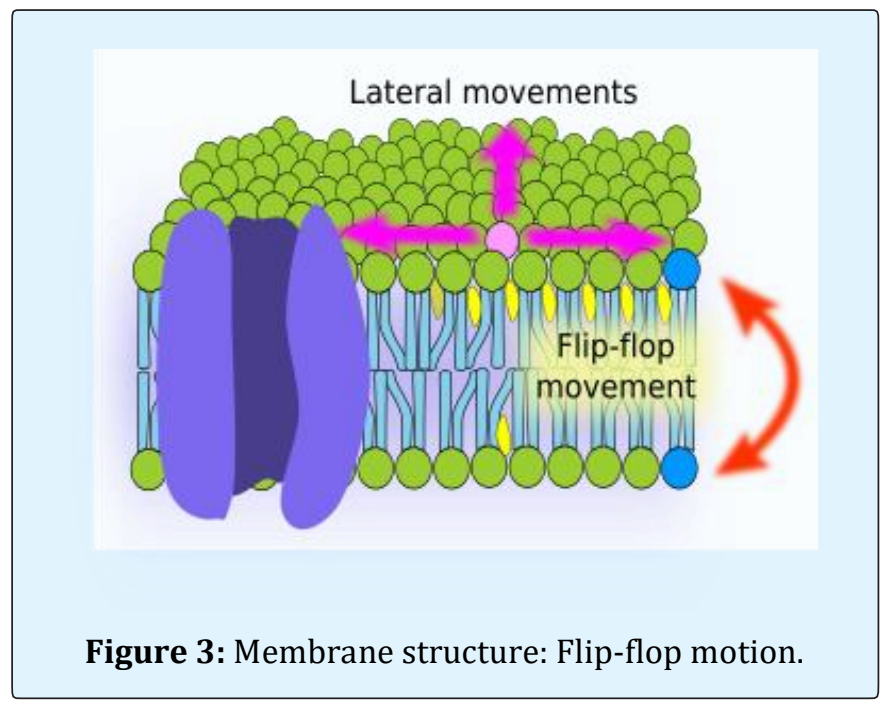

\section{Electrical Properties of Membranes}

\section{Membrane Capacitance}

Membranes are so thin, that they can separate charge on a sub microscopic scale. Negative charges accumulate at or near one surface of a membrane. These charges interact electrostatically over the short distance of membrane thickness with positive charges on the other side. The ability of membranes to accumulate and separate charges gives rise to the electric property which is termed as membrane capacitance. Capacitance increases in proportion to the dielectric constant of the material separating the charges and decreases with increasing distance between charges. Most membranes appear to contain a layer of lipid about $5 \mathrm{~nm}$ thick. If we assume that the lipid has a dielectric constant of 3 which is about that of an IS-carbon fatty acid, the membrane capacitance can be calculated to be about I microfarad per $\mathrm{cm} 2$. In a real cell, the ICF (intracellular fluid) and ECF (extracellular fluid) are the conductors and the lipid bilayer of the plasma membrane is insulating barrier [11].

\section{Membrane Resistance}

Membranes act as barriers to the free diffusion of various substances, including ions. Since electric current in an aqueous solution is a flow of ions, the relatively low mobility of ions through the lipid bilayer is equivalent to a high electric resistance. Membrane resistance is a measure of the ease with which ions move across the membrane under the influence of a potential difference [12].

\section{Equivalent Circuit for a Cell Membrane}

It is helpful to conceptualize the properties of capacitance and resistance in the form of an equivalent circuit in which a capacitor is wired in parallel with a resistor. The capacitor represents the major area of lipid bilayer which is impermeable to ions and the resistor represents the hydrophilic ion-permeable channels or carriers of the membrane. Electromotive force in the membrane bathing solution supplies the charge to the membrane capacitor [11,12].

\section{Response of Membrane Capacitance and Resistance to a Pulse of Current}

The electrical properties of the cell membrane can be examined by passing a pulse of current through the membrane. Consider a current of constant intensity, 11 (amperes), passed across the membrane from the electrode in the bath to the current electrode in the cell. This current is applied as a square pulse with Voltage pulse and flow through the wires, bath, electrodes and cell membrane. The high-ohm resistor has far greater resistance than the other resistances in the stimulating circuit. These properties were observed in patch-clamp recordings of cerebellar Purkinje neurons (in the presence of pharmacological blockers of other background ionic currents) and were more prominent in the sub-threshold region of the membrane potential [13]. 


\section{International Journal of Biochemistry \& Physiology}

This helps in maintaining the constancy of the stimulating current. The recording amplifier has a very high input resistance, preventing any appreciable current from leaving the cell through the recording electrode an abrupt onset. This current must pass through the membrane to make a complete circuit. While crossing the membrane, the current distributes itself between the parallel resistance and capacitance of the membrane. The time required for voltage to reach $63 \%$ of its asymptomatic value is proportional to both the resistance and the capacitance of the membrane. This is termed as the "time constant" of the membrane, which can explain a single electrical recording and the choice of an electric model for a biological system is based on complementary information (most commonly structural information) of the system investigated [14].

The current applied to the membrane flows initially through the capacitance of the membrane. As the capacitance charges (or discharges) exponentially, an increasing fraction of the total current passes through the resistive component of the membrane. After several time constants have elapsed, the potential closely approaches an asymptote and the applied current flows through the membrane resistance. When the applied current is terminated, the charge stored across the capacitor leaks through the resistor and the potential returns to the rest level with an exponential time course [15].

\section{Transport Processes Across the Cell Membrane}

Transport processes across the cell membrane are important due to the following reasons [16]:

i. They regulate cell volume and maintain the intracellular $\mathrm{pH}$ and ionic composition within a narrow range.

ii. They extract and concentrate metabolic fuels and building blocks from the environment and extrude toxic substances.

iii.They generate ionic gradients that are essential for the excitability of nerve and muscle.

\section{Classification of Transport Processes}

Various transport processes across the membrane can be classified as follows.

1. Non-mediated transport, i.e.. simple diffusion

2. Mediated transport

a. Facilitated diffusion. i.e.. carrier mediated passive transport

b. Active transport
- Primary transport (ATPase-linked pumps)

- Secondary transport (Gradient-coupled)

3. Vesicle mediated transport

a. Endocytosis

b. Exocytosis.

4. Antibiotic mediated ion transport.

\section{Simple Diffusion}

This means the molecular kinetic movement of molecules or ions through a membrane without the necessity of binding with carrier proteins in the membrane. The preferred direction is from the site of higher concentration to, the site of lower concentration of diffusing substance. Transfer of molecules across membranes by simple diffusion obeys Fick's law of diffusion which states that the flux of a substance through the membrane is due to the area of diffusion, the permeability of substance in the membrane, and the diffusion gradient between the two sites of the membrane.

The equation of Fick is further modified to include solubility of solute in the membrane.

\section{Factors Controlling Net Diffusion of Substances}

There is a concentration gradient across the membrane-solutes moving from high to low concentration. Also, there are electrical potential across the membrane-solutes move toward the solution that has opposite charges. Always, the permeability coefficient of the substance for the membrane allows the substances can across the membrane, and the hydrostatic pressure gradient across the membrane and the temperature increases particle motion.

\section{Osmosis}

It is a special case of diffusion. Water is transported across the membrane by osmosis. For osmosis two conditions must be satisfied. A solute must be present at a higher concentration on one side of the membrane than the other; and that solute must have a lower diffusional permeability across the membrane than for water. If there exists a difference in the concentration of water molecules on either side of a membrane there will be a net movement of water through the membrane at a rate determined by the water concentration gradient and the permeability coefficient of the membrane for water. 


\section{International Journal of Biochemistry \& Physiology}

\section{Facilitated Diffusion}

It is a diffusion facilitated by carrier protein not linked to metabolic energy. Carrier proteins are analogous to enzyme. Like enzymes they show saturation kinetics. As the enzyme undergo small conformational change after binding substrate. so do the carriers, i.e., they undergo structural change in the course of translocating solute/substrate across the membrane. Structurally similar molecules compete for carrier protein.

\section{An introduction to Medical Biophysics}

Effect of concentration of a substance on rate of diffusion through a membrane in which there is facilitated diffusion. The concentration at half-maximal velocity is equal to the binding constant $(\mathrm{Km})$ of the carrier for the solute transport substrate will decrease the transport rate of second substrate. This is analogous to competitive inhibition of an enzyme. For example, glucose, galactose, arabinose and 3-0-methylglucose compete for the same carrier like enzymes, carrier proteins are inhibited by poisons/inhibitors. Inhibitors bind carrier protein in a way that decreases its affinity for normal transport of substrate.

Carrier proteins can carry out a vector process, i.e., a directional process which delivers the solute from one side of the membrane to other [17]. The rate of facilitated diffusion depends on the below given factors. i. The concentration gradient across the membrane.

ii. The amount of carrier available.

iii. The rapacity of the solute/substrate-carrier interaction.

iv. The rapidity of the conformational change for both the loaded and unloaded carrier.

\section{Active Transport}

When the substance moves from the place of higher concentration to the place of lower concentration, then the process is known as downhill movement, i.e., diffusion. But the process of moving molecules uphill, i.e., against a concentration gradient is called active transport. It is of two types. In primary active transport the consumption of metabolic energy is coupled directly to the movement which is independent of the downhill movement of any other species. In secondary active transport the downhill movement of one species drives the uphill movement of another and metabolic energy is not utilized directly. This is termed as co-transport; if the movement are in opposite direction, it is termed countertransport [18].

\section{Primary Active Transport}

It is coupled directly to a continuous supply of energy. Energy comes from ATP or electrons flow down an electron transport chain. ATPase activity of carrier protein cleaves ATP to release energy. Different types of ATPase have been found to be associated with carrier protein molecules. These include $\mathrm{Na}+-\mathrm{K}^{+}$ATPase, $\mathrm{Ca}^{2+} \mathrm{ATPase}, \mathrm{H}^{ \pm} \mathrm{K}^{ \pm}$ATPase, and proton-ATPase. The most ubiquitous active transport mechanism in the body is the one that transports sodium out of cells to the exterior and at the same time pumps potassium ions from outside to the inside; it is called sodium-potassium pump. Both $\mathrm{Na}+-$ $\mathrm{K}+$ pump and $\mathrm{Na}^{ \pm} \mathrm{K}^{ \pm} \mathrm{ATPase}$ are tightly associated with the plasma membrane and are specifically inhibited by cardiotoxic steroids. $\mathrm{Na}^{ \pm} \mathrm{K}^{+}$ATPase (Figure 4) consists of two large subunits that span the membrane and two smaller glycoprotein subunits that are exposed to the outside of the cell membrane. The region of the protein that faces the cytoplasm has a site for binding ATP and three high-affinity sites for binding $\mathrm{Na}+$ ions. On the outer face the ATPase has two high-affinity binding sites for $\mathrm{K}^{+}$ ions as well as a binding site for oubain. Oubain or digitalis inhibits ATPase by binding to the extracellular domain [19]. For each revolution of the pump, one positive charge is moved from the interior of the cell to the exterior. This creates positivity outside the cell but leaves a deficit of positive ions inside the cell; that is, it causes negativity on the inside. Hence, this pump is electrogenic. The functions of $\mathrm{Na}^{ \pm} \mathrm{K}^{+}$pump are listed $[20,21]$. It maintains $\mathrm{Na}+$ and $\mathrm{K}+$ gradient required for propagation of nerve impulse. It maintains high intracellular $\mathrm{K}+$ required for various metabolic processes.

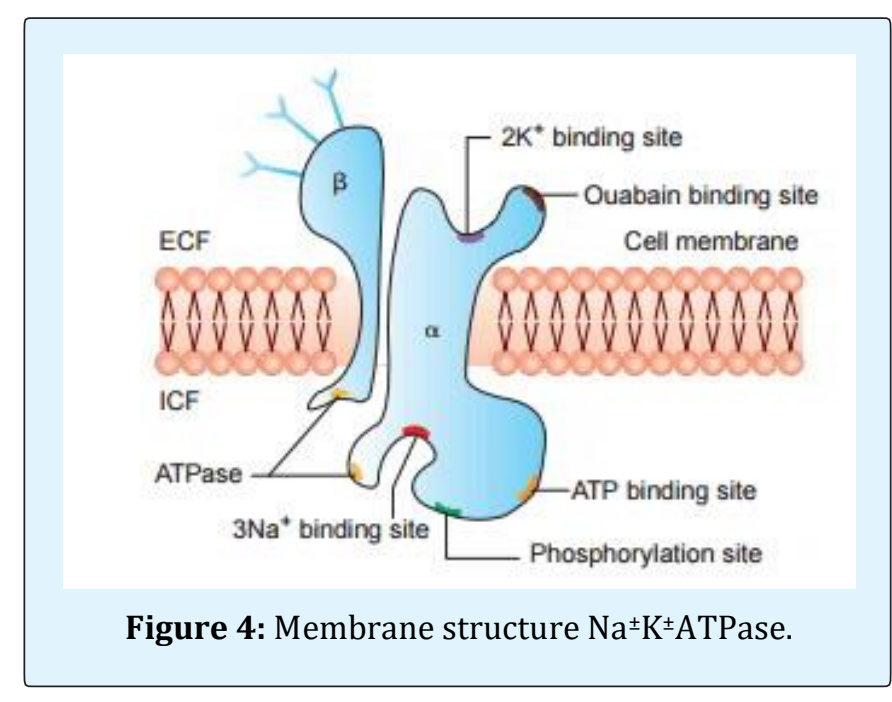




\section{International Journal of Biochemistry \& Physiology}

\section{Proton Mediated Transport}

Many bacterial transport systems are driven by the flow of protons across the plasma membrane. Driving force for this active transport of lactose is the proton motive force across the plasma membrane. i.e., it transport of a lactose molecule is coupled to the movement of a proton into the cell. Under physiological conditions, the proton gradient needed for this active transport is created by the flow of electrons from high potential donors (NADH) through respiratory chain. ATP dependent proton pump has been localized in the apical membrane of oxyntic cells.

\section{Secondary or Gradient Coupled Active Transport}

In this transport, active transport of metabolite is coupled with simultaneous movement of second substance which is moving down its concentration gradient. For example, glucose and amino acids are transported into some cells against very large concentration gradient, even though the carrier protein for the transport of these substances cannot cleave ATP to provide the required energy. Instead, the energy is provided by the concentration gradient of the sodium ions across the cell membrane. Here, the carrier protein has got two binding sites on its exterior side, one for sodium and one for glucose. Once both the sodium ion and glucose molecules are attached to the carrier protein, it undergoes conformational change and both sodium and glucose are transported to the inside of the cell. Calcium ions move against the concentration gradient, uphill from inside to outside. It is the energy gradient of the sodium ions which literally pulls the glucose to the interior causing its uphill transport. Since, glucose and sodium ions are moving in the same direction, this is called cotransport. If the concentration gradient of sodium is used for the transport of second substance uphill e.g., calcium ions, but in the opposite direction, then it is called counter transport. Common examples of co-transport are listed below:

i. Co-transport of sodium and sugars has been found in the proximal renal tubule, gallbladder and intestine.

ii. Co-transport of sodium and amino acids has been localised in intestine, proximal renal tubule, brain cells, choroid plexus leucocytes and ascites tumour cells.

iii. Disorders of sugar and amino acid co-transport system have been identified in certain hereditary diseases, e.g., Hartnup's disease.

\section{Free Energy Change During Simple and Facilitated Diffusion and Active Transport}

Simple diffusion and facilitated diffusion are spontaneous processes. Hence, the solute moves down a concentration gradient, i.e., until equilibrium is attained. The free energy change for these processes is negative because the solute becomes more randomly distributed. Thus, no input of energy is required for these two processes.

\section{Common Features of Facilitated Diffusion and Active Transport}

i. Both involve carrier protein and both show specificity for ions sugars and amino acids.

ii. Both resemble substrate-enzyme reaction, i.e., carrier protein' has a specific binding site.

iii. The carrier is saturable, so it has maximum rate of transport Vmax.

iv. The whole system has a binding constant $\mathrm{Km}$.

v. Structurally similar competitive inhibitors block transport.

\section{Endocytosis and Exocytosis}

They represent energy-dependent processes by which molecules of larger molecular weight are encapsulated in membrane as they enter or before they leave the cells. These mechanisms allow energy dependent transport of material between cells and the interstitial fluid. In endocytosis, invagination of the plasma membrane first encloses extracellular material and then seal, forming vacuoles or vesicles within the cell. These vacuoles or vesicles may fuse with Lysosomes where they and their contents are degraded. Pinocytosis and phagocytosis are the forms of endocytosis. Pinocytosis [22] refers to the endocytosis uptake of solutions into the cells ('cell drinking'). Phagocytosis refers to the engulfing of particulate matter, e.g., viruses and bacteria by neutrophils and macrophages ('cell eating') [23]. Exocytosis is a method by which most of the cells release macromolecules to the exterior. Here, macromolecules are released from cells into the extracellular fluid by fusion of intracellular vesicles with the plasma membrane. Examples of exocytosis include the release of peptide hormones from the endocrine glands and of neurotransmitters from nerve terminals. These processes are triggered by an influx of calcium ions in response to specific stimuli. A combination of endocytosis occurring at one membrane of a cell and exocytosis occurring at the other membrane is called transcytosis [24] (Figure 5). 


\section{International Journal of Biochemistry \& Physiology}

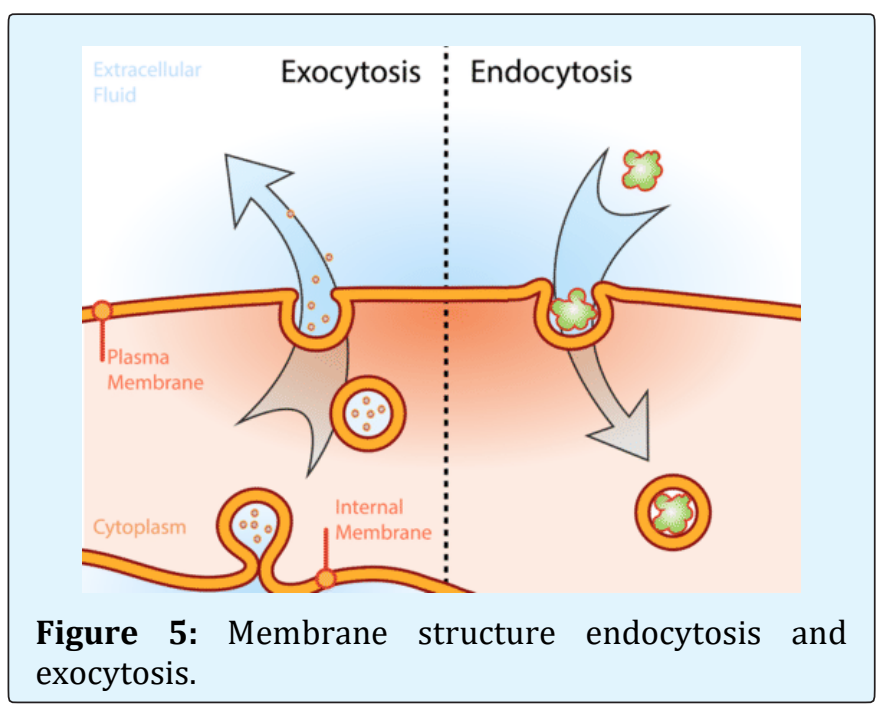

\section{Antibiotic Mediated Ion Transport}

Several fungal antibiotics specifically increase the permeability of biological membranes to certain cations. These antibiotics do not alter the specificity of a permease which is present in the membrane. But by them bring about ion transport, hence, are called ionophores. Two important ionophores are gramicidin A and valinomycin. Gramicidin A is an open-chain polypeptide consisting of fifteen amino acid residues. Because of hydrophobic nature, gramicidine A penetrates a lipid bilayer forming channel that traverse the membrane. Ion enters such a channel at one surface of the membrane [25].
Valinomycin on the other hand has a cyclic structure resembling a peptide chain containing 12 monomers. Basically, it is four-unit structure repeated three times. It specifically transports KT ions and no other ion. It increases KT conductance in virtually all membranes and lowers the energy barrier for transport of $\mathrm{K}$ across membranes. It is a donut-shaped molecule that binds a single metal ion in its central cavity. The hydrocarbon periphery enables the complex to traverse the hydrocarbon anterior of the membrane. Valinomycin can transport $\mathrm{K}+$ only above the phase transition temperature of the membrane, whereas cation transport by gramicidin A is insensitive to temperature. Valinomycin binds to an ion and behaves as mobile carrier but gramicidin simply provides a channel for the transport of $\mathrm{K}^{+}$[26].

\section{Antigens Bind to Receptors via Noncovalent Bonding Interactions}

The interactions between antigens and receptors of the immune system (Figure 6) are enhanced by simultaneous interactions between lymphocyteexpressed co-receptors and molecules on antigenpresenting cells or on complex antigens. Most receptorantigen interactions are multivalent, and this multivalence significantly increases the avidity of the receptor-antigen binding interaction. Binding of antigen to receptor induces a signalling cascade in the receptorbearing cell, which leads to alterations in the motility, adhesive properties, and transcriptional program of the activated cell. Antigen signalling is initiated in both $\mathrm{T}$ and $B$ cells by antigen-mediated receptor clustering.

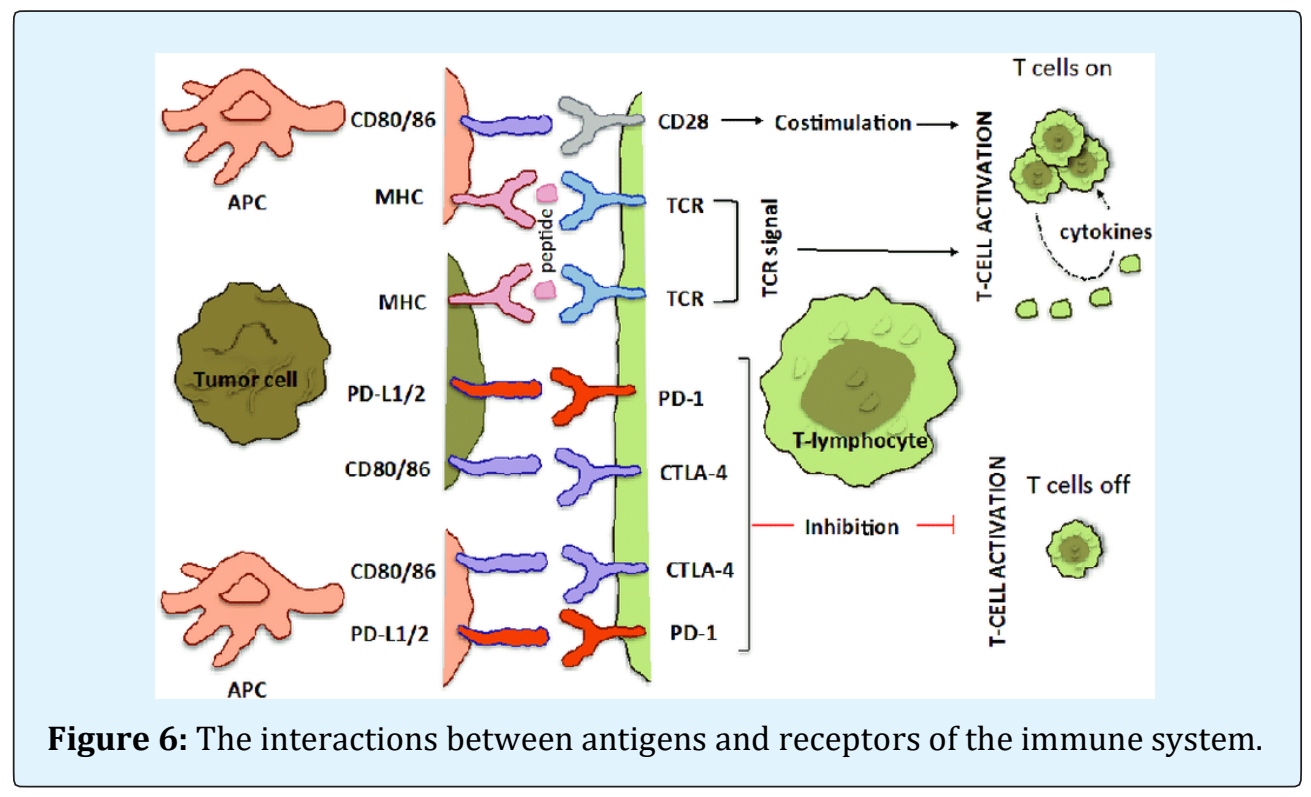

Santos Filho SD. New Trends in Immunology Membrane Biophysics. Int J Biochem Physiol 2019, 4(4): 000166. 


\section{International Journal of Biochemistry \& Physiology}

The clustered receptors are in specialized regions of the membranes called lipid rafts. The CD3 and Ig/Ig proteins, which are $\mathrm{T}$ - and B-cell receptor-associated signal transduction elements, are phosphorylated on Immunoreceptor tyrosine Activation Motifs (ITAMs), and these serve as docking points for adapter molecules. Downstream signalling enzymes and GEFs dock onto the adapter molecules and contact their substrates. These enzymes include phospholipase $\mathrm{C}$, which breaks down PIP2 into DAG and IP3. IP3 interaction with ER located receptors leads to release of intracellular calcium and activation of calcium-regulated proteins such as calcineurin phosphatase. Calcineurin dephosphorylates the transcription factor NFAT, allowing it access to the nucleus. DAG binds and protein kinase $\mathrm{C}$, leading eventually to NF-B activation. Docking of the adapter molecule Grb2 onto adapter proteins facilitates its binding to the GEF protein SOS and activation of the MAP kinase pathway, resulting in activation of the transcription factor AP-1 [27].

\section{The Antigen Receptor on B cells}

The type of antigen receptor present on B cells (Figure 7) is a membrane-bound form of the four-chain immunoglobulin molecule that the B cell secretes upon stimulation. An immunoglobulin molecule is commonly known as an antibody. Antibodies have two heavy and two light chains. The antibodies secreted by B cells upon stimulation are classified according to the amino acid sequence of the heavy chain, and antibodies of different classes perform different functions during an immune response. Antigen signalling in B cells proceeds according to signalling strategies shared among many cell types [28].

\section{The Antigen Receptor on T cells}

It is not an immunoglobulin molecule, although its protein domains are classified as belonging to the immunoglobulin superfamily of proteins. Most $\mathrm{T}$ cells bear receptors (Figures 7 and 8) made up of a heterodimer that recognizes a complex antigen, made up of a short peptide inserted into a groove on the surface of a protein encoded by the major histocompatibility complex (MHC) of genes. Some T cells bear receptors made up of receptor chains that recognize a different array of antigens. Antigen signalling in $\mathrm{T}$ cells shares many characteristics [29].

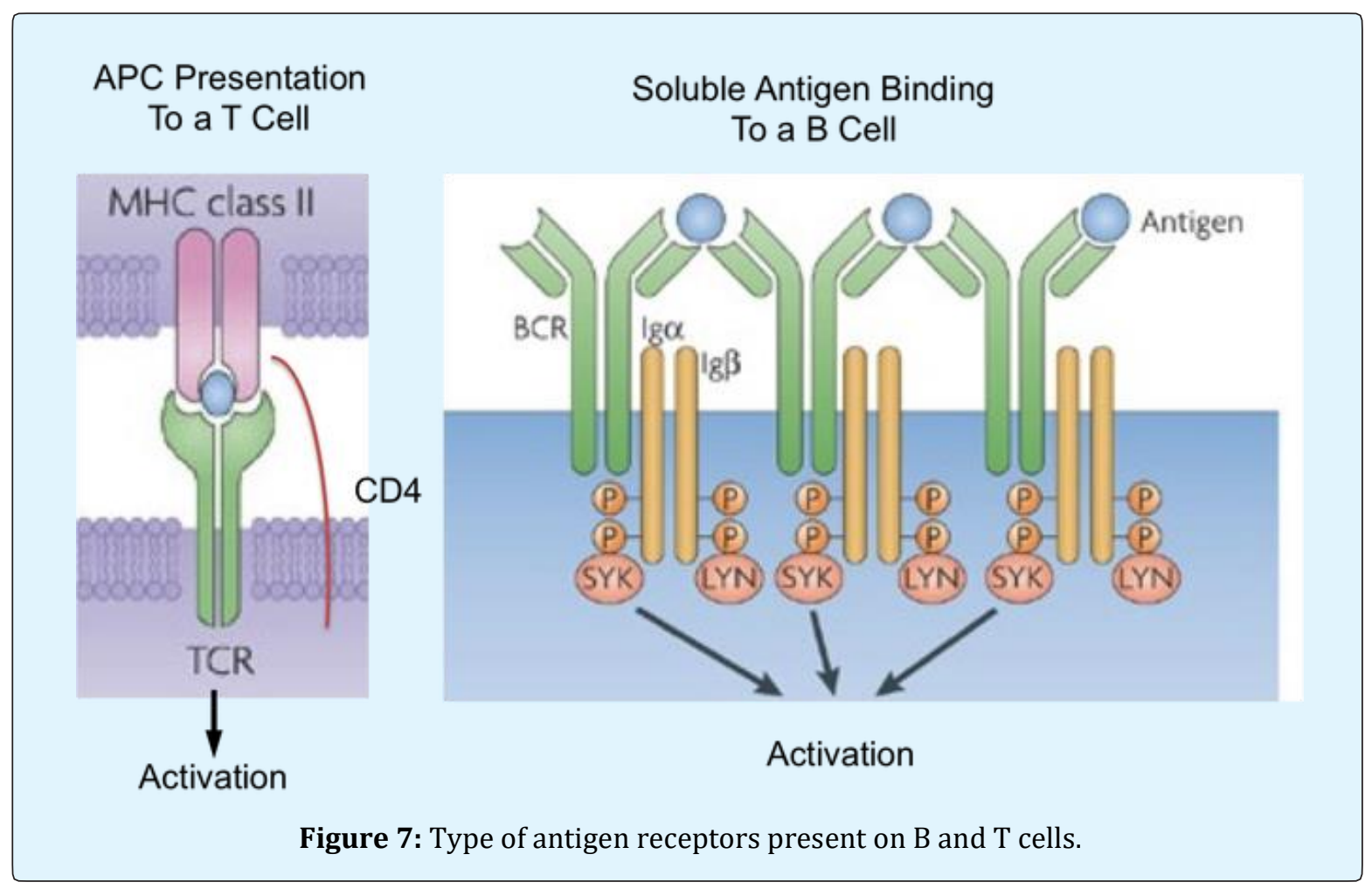

Santos Filho SD. New Trends in Immunology Membrane Biophysics. Int J Biochem Physiol 2019, 4(4): 000166. 


\section{International Journal of Biochemistry \& Physiology}

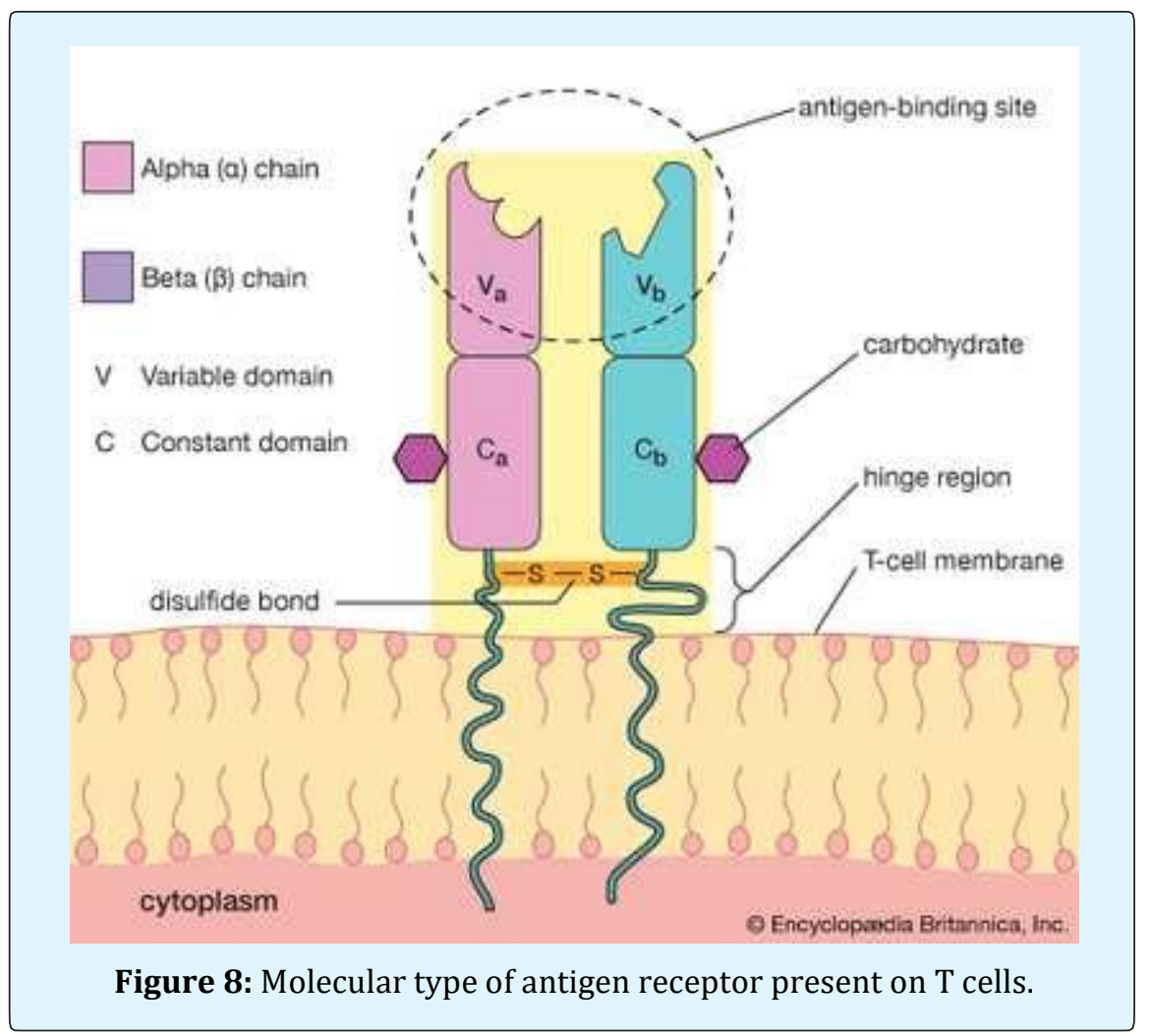

\section{References}

1. Lombard J (2014) Once upon a time the cell membranes: 175 years of cell boundary research. Biol Direct 9: 32 .

2. Kraft M (2013) Plasma membrane organization and function: moving past lipid rafts. Mol Biol Cell 24(8): 2765-2768.

3. Singer SJ, Nicolson GL (1972) The fluid mosaic model of the structure of cell membranes. Science 175(4023): 720-731.

4. Harder $T$ (2003) Formation of functional cell membrane domains: the interplay of lipid- and protein-mediated interactions. Phil Trans R Soc Lond B 358(1433): 863-868.

5. Hille B (1978) Ionic channels in excitable membranes. Current problems and biophysical approaches. Biophys J 22(2): 283-294.

6. Carrerall WA (2011) Voltage-Gated Calcium Channels. Cold Spring Harb Perspect Biol 3(8): a003947.

Santos Filho SD. New Trends in Immunology Membrane Biophysics. Int J Biochem Physiol 2019, 4(4): 000166.
7. Kuang Q, Purhonen P, Hebert H (2015) Structure of potassium channels. Cell Mol Life Sci 72: 3677-3693.

8. Shen Y, Song W, Ryan Barden D, Ren T, Lang C, et al. (2018) Achieving high permeability and enhanced selectivity for Angstrom-scale separations using artificial water channel membranes. Nature Comm 9(1): 2294.

9. Cruz-Chu ER, Malafeev A, Pajarskas T, Pivkin IV, Koumoutsakos P (2014) Structure and Response to Flow of the Glycocalyx Layer. Biophys J 106(1): 232243.

10. Fernández-Pérez EJ, Sepúlveda FJ, Peters C, Bascuñán D, Riffo-Lepe NO, et al. (2018) Effect of Cholesterol on Membrane Fluidity and Association of $A \beta$ Oligomers and Subsequent Neuronal Damage: A Double-Edged Sword. Front Aging Neurosci 10: 226.

11. Wang K, Zhao Y, Chen D, Fan B, Lu Y, et al. (2017) Specific membrane capacitance, cytoplasm conductivity and instantaneous young's modulus of single tumour cells. Sci Data 4: 170015. 


\section{International Journal of Biochemistry \& Physiology}

12. Akopian G, Barry J, Cepeda C, Levin MS (2016) Altered membrane properties and firing patterns of external globus pallidus neurons in the R6/2 mouse model of Huntington's disease. J Neurosci Res 94(12): 14001410.

13. Huang S, Hong S, De Schutter E (2015) Non-linear leak currents affect mammalian neuron physiology. Front Cell Neurosci 9: 432.

14. Mosgaard LD, Zecchi KA, Heimburg T, Budvytyte R (2015) The effect of the nonlinearity of the response of lipid membranes to voltage perturbations on the interpretation of their electrical properties. A new theoretical description. Membranes 5(4): 495-512.

15. Howell B, Medina LE, Grill WM (2015) Effects of Frequency-Dependent Membrane Capacitance on Neural Excitability. J Neural Eng 12(5): 056015.

16. Lodish H, Berk A, Zipursky SL (2000) Molecular Cell Biology. $4^{\text {th }}$ (Edn.), New York: WH Freeman.

17. The International Transport Consortium (ITC) (2010) Membrane transportes in drug development. Nat Ver Drug Discov 9(3): 215-236.

18. Giladi M, Shor R, Lisnyansky M, Khananshvili D (2016) Structure-Functional Basis of Ion Transport in Sodium-Calcium Exchanger (NCX) Proteins. Int J Mol Sci 17(11): 1949.

19. Hamlyn JM, Blaustein MP (2016) Endogenous Ouabain: Recent Advances and Controversies. Hypertension 68(3): 526-532.

20. Clausen MV, Hilbers F, Poulsen H (2017) The Structure and Function of the Na,K-ATPase Isoforms in Health and Disease. Front Physiol 8: 371.
21. Yan Y, Shapiro JI (2016) The physiological and clinical importance of sodium potassium ATPase in cardiovascular diseases. Curr Opin Pharmacol 27: 4349.

22. Canton J (2018) Macropinocytosis: New Insights into Its Underappreciated Role in Innate Immune Cell Surveillance. Front Immunol 9: 2286.

23. Morrissey MA, Williamson AP, Steinbach AM, Roberts EW, Kern N, et al. (2018) Chimeric antigen receptors that trigger phagocytosis. eLife 7: e36688.

24. Mayor S, Parton RG, Donaldson JG (2014) ClathrinIndependent Pathways of Endocytosis. Cold Spring Harb Perspect Biol 6(6): a016758.

25. Ghai I, Ghai S (2018) Understanding antibiotic resistance via outer membrane permeability. Infect Drug Resist 11: 523-530.

26. Tempelaars $\mathrm{MH}$, Rodrigues $\mathrm{S}$, Abee $\mathrm{T}$ (2011) Comparative Analysis of Antimicrobial Activities of Valinomycin and Cereulide, the Bacillus cereus Emetic Toxin. Appl Environ Microbiol 77(8): 27552762.

27. Choe W, Durgannavar TA, Chung SJ (2016) Fc-Binding Ligands of Immunoglobulin G: An Overview of High Affinity Proteins and Peptides. Materials (Basel) 9(12): 994.

28. Yam-Puc JC, Zhang L, Zhang Y, Toellner KM (2018) Role of B-Cell receptors for B-Cell development and antigen-induced differentiation. F1000Res 7: 429.

29. Lemke H (2018) Immune Response Regulation by Antigen Receptors' Clone-Specific Nonself Parts. Front Immunol 9: 1471. 